\title{
Prolyl-tRNA synthetase inhibition reduces microsporidia infection intensity in honey bees
}

\author{
Jonathan W. SNOw \\ Department of Biology, Barnard College, New York, NY 10027, USA \\ Received 8 March 2019 - Revised 13 October 2019 - Accepted 22 January 2020
}

\begin{abstract}
Nosema ceranae is a microsporidian parasite that is pathogenic to honey bees, causing disease at the individual and colony level. N. ceranae infection can be controlled by treatment with Fumagillin, but as this the future of this drug is uncertain, alternative treatment strategies are critical. Genomic examination of proteostasis pathways in $N$. ceranae and its host, Apis mellifera, has revealed key differences in component conservation. One example is the amino acid response (AAR), which responds to amino acid limitation through the sensing of uncharged tRNA molecules. The AAR is conserved in the honey bee, but not in $N$. ceranae. Pharmacological inhibition of prolyl-tRNA synthetase activity resulted in a substantial reduction in $N$. ceranae infection intensity. Evident toxicity to bees suggests further work is required to make this approach safe and feasible. However, these results provide proof of principle that tRNA synthetase inhibition could be an effective future treatment strategy.
\end{abstract}

\section{Nosema ceranae / Microsporidia / Honey bee / Infection / tRNA synthetase}

\section{INTRODUCTION}

Colonies of the western honey bee, Apis mellifera, have suffered from increased mortality in recent years that is likely caused by a complex set of interacting stresses (Goulson et al. 2015). There has been intensifying focus on the role of microbial attack on honey bee health (Evans and Schwarz 2011). The microsporidian species Nosema ceranae and Nosema apis can cause individual mortality in honey bees and have been implicated in colony disease (Weiss and Becnel 2014; Goblirsch 2017; Martín-Hernández et al. 2018). N. ceranae is now one of the most common pathogens of the honey bee which infects the

Electronic supplementary material The online version of this article (https://doi.org/10.1007/s13592-020-00742-9) contains supplementary material, which is available to authorized users.

Corresponding author: J. Snow, jsnow@barnard.eduW. Snow, jsnow@barnard.edu

Manuscript editor: Peter Rosenkranz midgut and causes energetic stress, epithelial damage, and, when untreated, death.

$N$. ceranae infection has traditionally been controlled by treatment with the drug Fumagillin, a methionine aminopeptidase 2 inhibitor (reviewed in (van den Heever et al. 2014)). Yet, the effectiveness of Fumagillin treatment in controlling $N$. ceranae at the colony level appears limited in scope and duration (Mendoza et al. 2017). High doses of this drug impact host cell function, and evidence suggests that $N$. ceranae can evade suppression in some circumstances (Huang et al. 2013). Most critically, this drug was temprarily unavailable due to production problems, leading to doubts about its future. Thus, efforts to find alternative treatment strategies are critical to protect honey bees from this parasite (van den Heever et al. 2014; Holt and Grozinger 2016).

As comparative genomics indicates that microsporidia have lost many of the cellular processes and pathways found in free-living 
eukaryotes (Nakjang et al. 2013), it appears likely that these organisms could possess fewer genes encoding proteins involved in protecting cells from stress. Examination of proteostasis pathways in Nosema species and its host revealed key differences in component conservation and function. One such proteostasis pathway is the amino acid response (AAR) activated by amino acid deprivation. Genome analysis revealed that the components of the AAR are conserved in the honey bee, but not in Nosema species, suggesting that stresses that trigger this pathway might provide an attractive therapeutic strategy. Pharmacologic inhibition of aminoacyl-tRNA synthetases (AARSs), which catalyze the attachment of the appropriate amino acid to its cognate tRNA to form an aminoacyl-tRNA, cause proteotoxic stress, and activate the AAR. Recent discoveries have prompted a significant interest in targeting tRNA synthetases in a wide variety of eukaryotic infectious organisms that cause disease in humans and livestock (Pham et al. 2014). Most notably, Halofuginone inhibits prolyl-tRNA synthetase activity in malaria-causing parasites (Khan 2016). Comparison of the honey bee bifunctional glutamyl/prolyl-tRNA synthetase (EPRS) and the Nosema spp. prolyl-tRNA synthetase (PRS) with homologs known to be inhibited by Halofuginone suggested that they would be inhibited by this drug as well. The impacts of prolyl-tRNA synthetase inhibition on $N$. ceranae infection and host function and survival were evaluated.

\section{MATERIALS AND METHODS}

\subsection{Honey bee tissue collection}

Honey bees were collected from outbred colonies consisting of a typical mix of Apis mellifera subspecies found in North America at different times during the months of April-October in New York, New York. Only visibly healthy bees were collected, and all source colonies were visually inspected for symptoms of common bacterial, fungal, and viral diseases of honey bees. For most experiments, bees were collected from the landing board of the colony with the entrance blocked and likely represent primarily foragers. Gut tissue was removed from abdomens, and midguts were dissected.

\subsection{Ortholog screening}

AAR pathway and aminoacyl-tRNAsynthetase gene candidates from Drosophila melanogaster and Caenorhabditis elegans were used to find orthologs in the honey bee genome using the BLAST family of search functions (www.ncbi.nlm.nih.gov). AAR pathway and aminoacyl-tRNA-synthetase gene candidates from Saccharomyces cerevisiae were used to find orthologs in the N. ceranae as well as other available microsporidian genomes using the same methods. The KEGG (Kyoto Encyclopedia of Genes and Genomes) database was also used as a guide for comparing pathways between species (Kanehisa and Goto 2000).

\subsection{Caging and chemical treatments}

For caging experiments, honey bees were selected as above and kept in 177.4-mL (6 oz.) square-bottomed Drosophila stock bottles (VWR) with modified foam tube plugs (Jaece Industries). Approximately 15 bees were added to each cage, and cages were maintained in incubators at $35^{\circ} \mathrm{C}$ in the presence of PseudoQueen (Contech, Victoria, British Columbia, Canada) as a source of queen mandibular phermone (QMP). Bees were fed $33 \%$ sucrose via a modified $1.5-\mathrm{ml}$ screw-cap tube and allowed to feed ad libitum with or without Halofuginone (Sigma), Febrifugine (Sigma), or Fumagillin-B (Medivet) as noted in the text. When DMSO was used as the chemical solute, equivalent amounts of DMSO were added to the food of the control group. No differences in the amount of food consumed per bee were observed in any treatment group.

\subsubsection{N. ceranae infection}

Infection intensity was quantified by counting spore number per bee using light microscopy (Cantwell 1970). N. ceranae spores for infecting bees were obtained from infected individuals. In this case, midguts from infected or uninfected bees were individually crushed in $0.5-\mathrm{ml} \mathrm{H} 20$ 
and washed 3 times with water and resuspended in $33 \%$ sucrose syrup at a concentration of $1 \times 10^{6}$ spores per $\mathrm{ml}$. Bees were allowed to consume food ad libitum for $24 \mathrm{~h}$ before food was replaced with $33 \%$ sucrose syrup alone. Bees in the uninfected group always received sucrose syrup containing a midgut from an uninfected bee processed in the same way as the midgut containing spores.

\subsubsection{Newborn caging, infecting, and chemical treatments}

When newly emerged bees were used, they were collected after hatching from a capped brood frame overnight in an incubator at $35{ }^{\circ} \mathrm{C}$ in the presence of PseudoQueen (Contech, Victoria, British Columbia, Canada) as a source of queen mandibular phermone (QMP). Approximately 30 newly eclosed bees were placed in each $4.8 \times 3.4 \times 8.4$-in. acrylic cage with sliding door machined at Carleton Labs, Columbia University, New York, NY, USA. Approximately 4 foragers from the same source colony (marked with a spot of paint (Testors, Vernon Hills, IL, USA)) were added to each cage. Bees were fed sucrose solution and supplied with a $\sim 5$-g pollen substitute patty (1:1 mix of BeePro and sucrose solution). On day 2 post-eclosion, $N$. ceranae spores $\left(5 \times 10^{6} / \mathrm{ml}\right)$ were fed to bees in sucrose solution ad libitum (Fries et al. 2013) for $48 \mathrm{~h}$. At 3-day postinfection, honey bees were fed sucrose solution containing one of the pharmacologic agents or vehicle control alone at the indicated doses. After 4 days of drug feeding, honey bee midguts were dissected, pooled, and crushed in $0.5-\mathrm{ml}$ water/bee, and the number of mature spores was counted by light microscopy as previously described (Fries et al. 2013). Survival during drug treatment was monitored in parallel.

\subsection{RNA isolation, reverse transcription, and quantitative PCR gene expression}

RNA was prepared from bees from the described populations as before (Johnston et al. 2016). Briefly, the midgut was manually crushed with a disposable pestle in TRIzol Reagent
(Invitrogen, San Diego, CA). The RNA was extracted as per the manufacturer's instructions, and contaminating DNA was removed through treatment with RQ1 RNase-Free DNase (Promega, Madison, WI). RNA was then quantified, and cDNA was synthesized using the iScript cDNA Synthesis Kit (Bio-Rad, Hercules, CA). Subsequently, $1 \mu$ of cDNA was then used as a template for quantitative PCR to determine the transcript levels for genes of interest using the iQ SYBR Green Supermix (Bio-Rad, Hercules, CA). Primer sequences for transcripts of honey bee genes of interest developed for this study are lactate dehydrogenase (F: 5'-AAACAGAAGGCTGG GAGCAG-3', R: 5'-ACGGTGACTTTGTG CGTACT-3') and asparaginyl-tRNA synthetase (F: 5'-CCTGGTGGTGCAGATTCCAT-3', R: 5'ATACATCGCCCATTGCTGGA-3'). For honey bee genes, the difference between the threshold cycle $(\mathrm{Ct}$ ) number for honey bee $\beta$-actin (primer sequence from (Johnston et al. 2016) and that of the gene of interest was used to calculate expression levels relative to $\beta$-actin using the $\Delta \Delta \mathrm{C}_{\mathrm{T}}$ method.

\subsection{DNA extraction and quantitative PCR for infection quantitation}

DNA extraction was performed using a modified Smash \& Grab DNA Miniprep protocol (Rose et al. 1990). Subsequently, $1 \mu$ l of DNA was used as a template for quantitative PCR to determine the levels of infection for Nosema sp. using the iQ SYBR Green Supermix (Bio-Rad, Hercules, CA) in a LightCycler 480 thermal cycler (Roche, Branchburg, NJ). Primer sequences for $N$. ceranae $\beta$-actin and honey bee ATP synthase F1 subunit alpha (ATP5a) gene were from Snow et al. (2019). The ATP5a gene measures honey bee genome equivalents by amplifying only genomic DNA. The difference between the threshold cycle $(\mathrm{Ct})$ number for honey be ATP5a and $N$. ceranae $\beta$-actin was used to calculate the level infection relative to ATP5a using the $\Delta \Delta \mathrm{C}_{\mathrm{T}}$ method. A sample was considered negative for a specific Nosema species if it did not amplify any product by 35 cycles, and zero was entered as the value in these cases. 


\subsection{Statistical analysis}

Data is presented as means \pm SEM shown. For gene expression analysis, data was $\log 10$ transformed. For two groups, data was compared using unpaired $t$ tests with Welch's correction when values fit normal distributions or Mann-Whitney U nonparametric tests when they did not fit normal distributions. Normality was assessed using Shapiro-Wilk tests. When more than two groups were being compared, data was compared using one-way ANOVA with Tukey's multiple comparison test when values fit normal distributions or a KruskalWallis test when they did not. For survival analysis, treated versus untreated groups were compared using the Gehan-Breslow-Wilcoxon test.

\section{RESULTS}

AAR pathway components are conserved in the honey bee, but not Nosema ceranae or other microsporidiaWe previously showed that the
AAR pathway (Figure 1 and Suppl Figure 1) is conserved in honey bees (Johnston et al. 2016) and Suppl Table 1. However, similar analysis revealed that $N$. ceranae does not appear to possess genes encoding general control nonderepressible 2 (GCN2) or activating transcription factor 4 (ATF4) (general control nonderepressible 4 (GCN4) in yeast) homologs. In addition, no other microsporidia have either of these AAR components. All three subunits of eIF2 are found in N. ceranae (Suppl Table 2), and alignment of the alpha subunit from $N$. ceranae with that of $S$. cerevisiae shows that Ser51 is conserved, suggesting that eIF2 regulation by phosphorylation is possible (Suppl Figure 2).

Apis mellifera bifunctional glutamyl/prolyl-tRNA synthetase and $N$. ceranae prolyl-tRNA synthetase predicted to bind HalofuginoneHalofuginone, a well-characterized inhibitor of prolyl-tRNA synthetase activity, was used to trigger the AAR in both species. In unicellular eukaryotes such as microsporidia (Lee et al. 2010), the ERS and

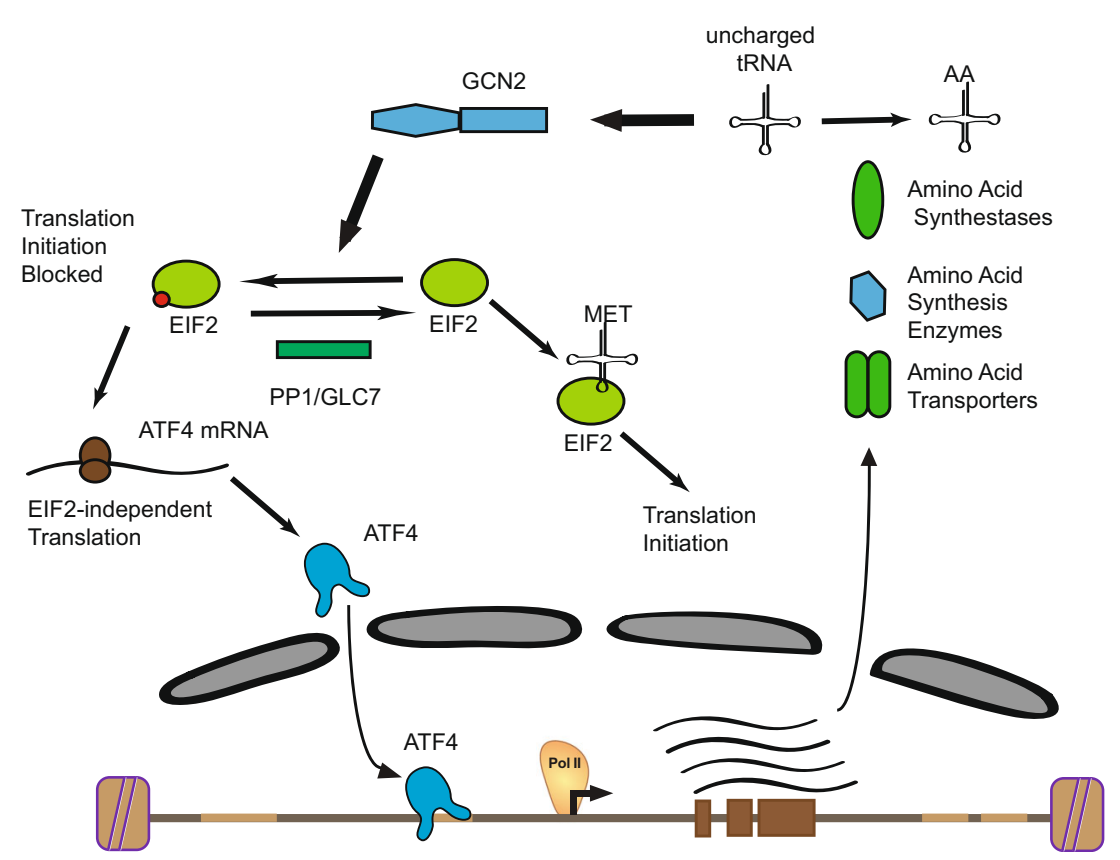

Figure 1. Honey bee and $N$. ceranae amino acid response (AAR) pathway. Diagram of components of the AAR pathway conserved in honey bees (blue) and both honey bees and Nosema spp. (green). 
PRS activities exist in two proteins, while in higher eukaryotes, such as the honey bee, these functions have been combined in a single fusion protein (Guo et al. 2010). Two recent studies showed the key amino acids involved in binding of Halofuginone to Plasmodium prolyl-tRNA synthetase (PRS) and its inhibition (Jain et al. 2015; Herman et al. 2015). The bifunctional glutamyl/prolyl-tRNA synthetase (EPRS) protein sequence for the honey bee (XP_026301491.1) and the prolyl-tRNA synthetase (PRS) sequence for $N$. ceranae (XP_024331292.1) were identified (Figure 2a). Regions containing critical Halofuginone binding site for the honey bee EPRS and the $N$. ceranae PRS proteins were aligned with the corresponding regions from the PRS proteins of Plasmodium falciparum and
$S$. cerevisiae (Figure $2 \mathrm{~b}$ ). Based on this comparison, both honey bee and $N$. ceranae proteins are predicted to bind Halofuginone (critical amino acid residues highlighted in red). In addition, both have a threonine in position 512 (numbering based on $P f$ cPRS) instead of the mutation to serine that is thought to make $S$. cerevisiae unaffected by Halofuginone (residue highlighted in orange). Examination of the PRS sequences for other microsporidia with available genomes (Suppl Table 3) through sequence alignment suggests that most species are sensitive to Halofuginone inhibition (data not shown).

Halofuginone controls existing infections by $N$. ceranae in experimentally and naturally infected beesTo determine how PRS inhibition would

\section{a}

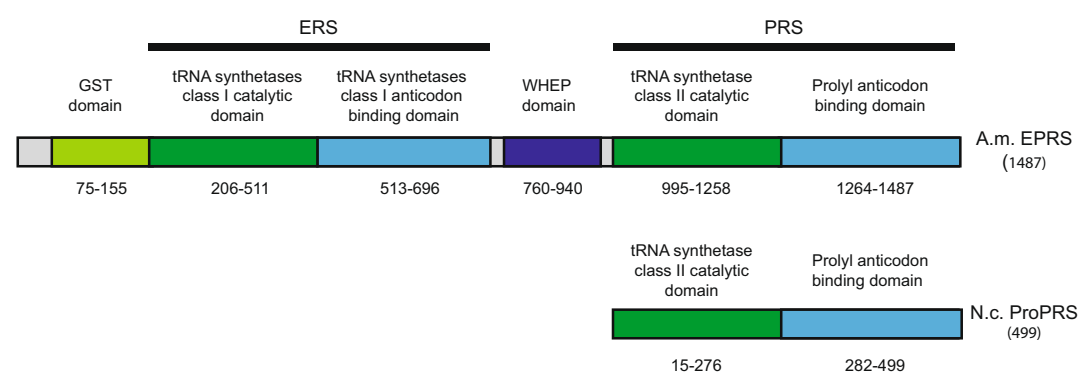

b
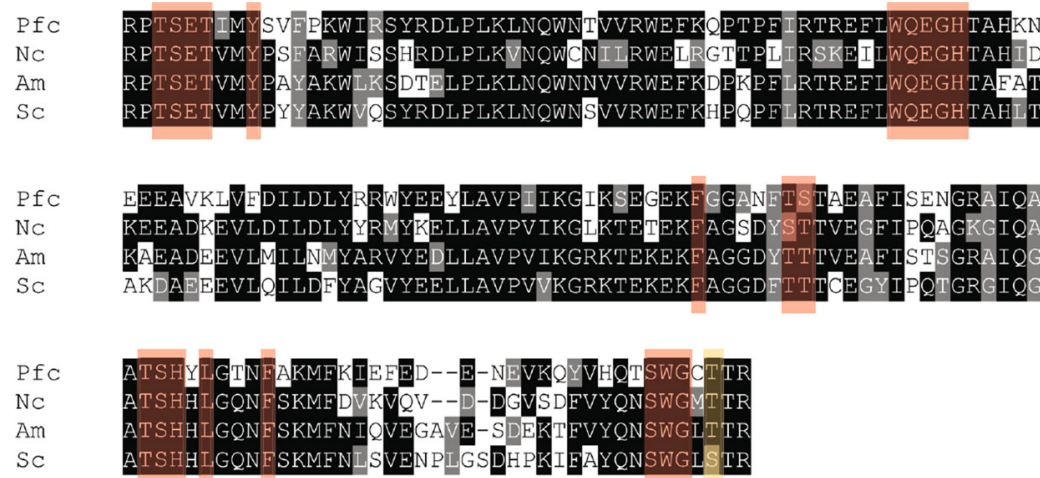

Figure 2. Apis mellifera bifunctional glutamyl/prolyl-tRNA synthetase and $N$. ceranae prolyl-tRNA synthetase predicted to bind Halofuginone. A schematic representation of the domain structures of the bifunctional glutamyl/ prolyl-tRNA synthetase (EPRS) protein of the honey bee (XP_016772424.1) and the prolyl-tRNA synthetase (PRS) protein for N. ceranae (XP_002996195.1) (a). Sequence alignment of the region containing critical Halofuginone binding site for the honey bee EPRS and the $N$. ceranae PRS protein with the corresponding regions from the PRS proteins of Plasmodium falciparum and Saccharomyces cerevisiae (b ). Critical amino acids are shaded in red while the resistance mutation found in S. cerevisiae is shaded in yellow. 
impact $N$. ceranae infection in honey bees, experimentally infected bees were fed either sucrose syrup or sucrose syrup containing $200-\mu \mathrm{M}$ Halofuginone for 2 days starting on 6-day postinfection. On day 8 postinfection, spore levels were measured using light microscopy to determine the effects of PRS inhibition on N. ceranae infection intensity. Feeding infected bees Halofuginone or the canonical anti-Nosema agent Fumagillin for $48 \mathrm{~h}$ both resulted in a reduction in infection intensity in infected bees (Figure 3a). Similar results were observed for bees captured on the landing board of a heavily infected colony (80\% prevalence) (Figure 3b). As spore counts do not quantify all life stages of microsporidia, the amounts of
$N$. ceranae $\beta$-actin gene relative to honey bee ATP5a gene by qPCR were also used to assess relative infection intensity. Similar reductions in infection intensity were found through measuring spore counts or genome equivalents (Suppl Figure 3). There were no decreases in survival to bees over this 2-day treatment. To assess the impacts of Halofuginone treatment on honey bees, uninfected bees from the landing board were fed various concentrations of Halofuginone ( 40 or $200 \mu \mathrm{M}$ ), vehicle control, or Fumagillin. Honey bees had reduced survival by 5 days after starting treatment with $200-\mu \mathrm{M}$ Halofuginone $(p<0.01)$ (the dose required to reduce $N$. ceranae infection) (Figure 3c). a

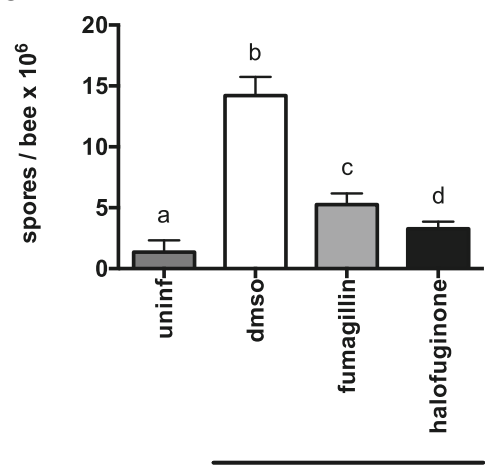

infected b

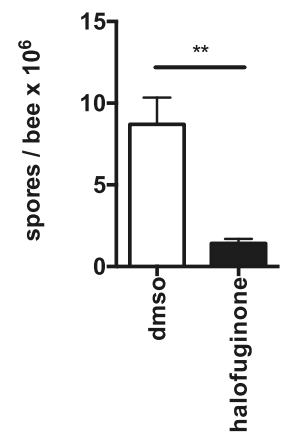

C

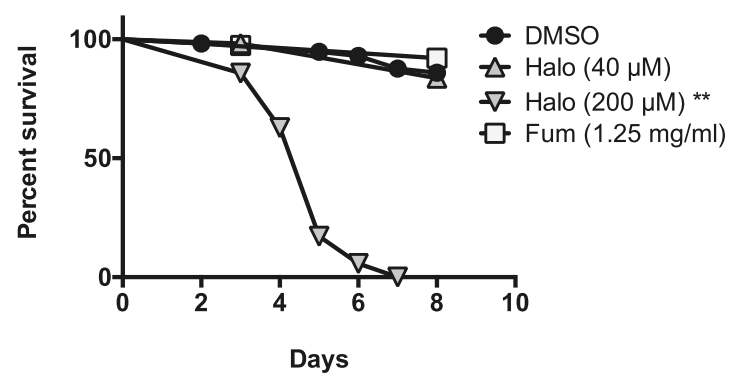

Figure 3. Prolyl-tRNA synthetase inhibition by Halofuginone reduces $N$. ceranae infection intensity in experimentally or naturally infected honey bees. $N$. ceranae levels in midguts as determined by spore count using light microscopy 8 days postinfection in uninfected or infected bees fed sucrose syrup containing DMSO or Halofuginone for the final 2 days $\mathrm{a} \neq \mathrm{b} \neq \mathrm{c} \neq \mathrm{d}, p<0.05$ (a). N. ceranae levels as determined by spore count using light microscopy in individual bees from an infected colony fed sucrose syrup containing DMSO, Fumagillin, or Halofuginone for 2 days $* * p<0.01$ (b). Survival of individual uninfected honey bees fed sugar solution containing no drug $(n=58)$, $1.25-\mathrm{mg} / \mathrm{ml}$ Fumagillin $(n=39)$, or Halofuginone $(40 \mu \mathrm{M}(n=55)$ or $200 \mu \mathrm{M}(n=35)) * * p<0.01(\mathbf{c})$. 
Febrifugine controls existing infections by $N$. ceranae in experimentally and naturally infected beesHalofuginone is the halogenated derivative of
Febrifugine (Figure 4a), the biologically active antimalarial found in the Dichroa febrifuga herb (Jiang et al. 2005). To determine if PRS inhibition a<smiles>O=C(CC1NCCCC1O)Cn1cnc2ccccc2c1=O</smiles>

febrifugine<smiles>O=C(C[C@@H]1NCCC[C@H]1O)Cn1cnc2cc(Br)c(Cl)cc2c1=O</smiles>

halofuginone

\section{C}

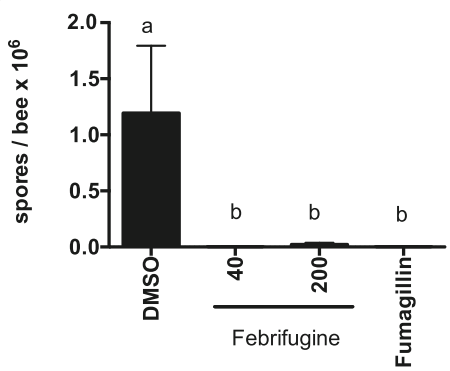

e

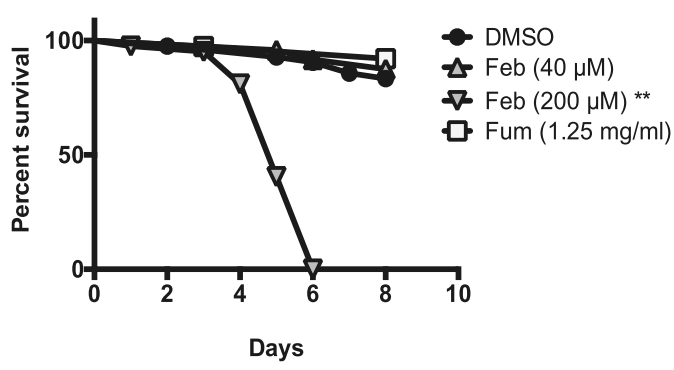

b

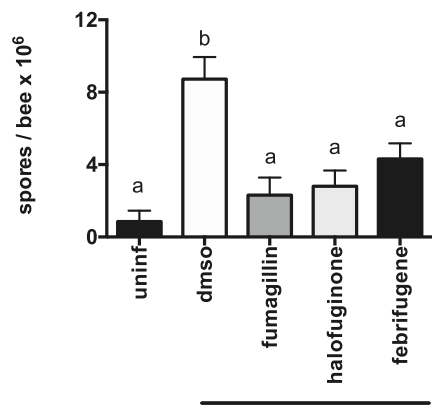

infected

d

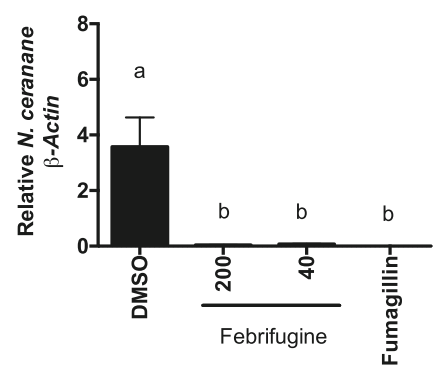

$\mathbf{f}$

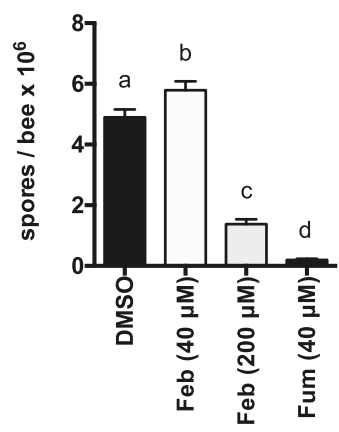

Figure 4. Prolyl-tRNA synthetase inhibition by Febrifugine reduces $N$. ceranae infection intensity in experimentally or naturally infected honey bees. Chemical structures of Halofuginone and Febrifugine (a). N. ceranae levels in midguts of newly eclosed bees as determined by spore count using light microscopy 8-day postinfection in uninfected or infected bees fed sucrose syrup containing DMSO, Fumagillin, Halofuginone, or Febrifugine for the final 2 days $\mathrm{a} \neq \mathrm{b}, p<0.05$ (b). $N$. ceranae levels in midguts as determined by spore count using light microscopy (c) or by qPCR (d) 8-day postinfection in uninfected or infected bees fed sucrose syrup containing DMSO, Fumagillin, or Febrifugine for 5 days $\mathrm{a} \neq \mathrm{b}, p<0.05$. Survival of individual honey bees fed sugar solution containing no drug $(n=42), 1.25-\mathrm{mg} / \mathrm{ml}$ Fumagillin $(n=38)$, or Febrifugine $(40 \mu \mathrm{M}(n=25)$ or $200 \mu \mathrm{M}(n=42)) * * p<0.01$ (e). N. ceranae levels in midguts as determined by spore count using light microscopy 11-day postinfection in uninfected or infected bees fed sucrose syrup containing DMSO, Fumagillin, or Febrifugine for 4 days $a \neq b$, $p<0.05$ (f). 
by this naturally occurring compound would impact $N$. ceranae infection in honey bees, experimentally infected bees were fed either sucrose syrup or sucrose syrup containing $200-\mu \mathrm{M}$ Febrifugine for 2 days starting on 6-day postinfection. On day 8 postinfection, spore levels were measured using light microscopy to determine the effects of PRS inhibition on $N$. ceranae infection intensity. Feeding infected bees Febrifugine for $48 \mathrm{~h}$ resulted in reduction in infection intensity in infected bees by spore count (Figure 4b). Experimentally infected bees were fed either sucrose syrup or sucrose syrup containing $200-\mu \mathrm{M}$ or 40 $\mu \mathrm{M}$ Febrifugine for 5 days starting on 3-day postinfection. Longer treatment regimens resulted in almost complete ablation of $N$. ceranae in those bees fed either Febrifugine (40 or $200 \mu \mathrm{M})$ or Fumagillin (1.25 mg per $\mathrm{ml}$ ) relative to bees fed vehicle control (DMSO) by spore count (Figure $4 \mathrm{c}$ ) or genome equivalents (Figure $4 \mathrm{~d}$ ). To assess the impacts of Febrifugine treatment on honey bees, uninfected bees were gathered from the landing board and fed them Febrifugine ( 40 or $200 \mu \mathrm{M}$ ), vehicle control, or Fumagillin. Here, it was found that honey bees had reduced survival by 5 days after starting treatment with $200-\mu \mathrm{M}$ Febrifugine $(p<0.01)$, but no change in survival over the 8-day time course using treatment with $40-\mu \mathrm{M}$ Febrifugine (Figure 4e).

The impact of Febrifugine treatment on infected of age-matched, newly eclosed bees was also assessed. Newly emerged bees were infected on day 3 post-eclosion and then fed Febrifugine (40 or $200 \mu \mathrm{M})$ or Fumagillin $(40 \mu \mathrm{M})$ or vehicle control for 4days starting on day 7 post-eclosion. In these newly eclosed bees, honey bees only had reduced infection intensity as assessed by spore count when treated with the higher dose of Febrifugine (Figure 4f). There were no differences in survival between groups over the 4 days of treatment (Suppl Figure 3c).

\section{Robust AAR induction after Halofuginone and} Febrifugine treatment in the honey beeTo determine whether inhibition of prolyl-tRNA synthetase activity would result in induction of the AAR in the honey bee, the expression of putative target genes was examined. As transcriptional activation by the transcription factor ATF4 is the primary mechanism regulating AAR-induced gene expression (Figure 2 and Suppl Figure 1), genes that have been shown to be dependent on ATF4 after its activation by the related unfolded protein response (UPR) pathway in the fruit fly (Lee et al. 2015) were tested. The glucose metabolism enzyme lactate dehydrogenase ( $L d h$ ) (Figure 5a) and the asparaginyl-tRNA synthetase gene $(N A R S)$ (Figure 5b) were upregulated significantly in the honey bee digestive tract after $48 \mathrm{~h}$ of Halofuginone treatment relative to bees consuming sucrose syrup alone, while the level of the reference gene $(\beta$-actin ) was not altered by treatment (Figure 5c). After Febrifugine treatment, $L d h$ was upregulated significantly in the honey bee digestive tract after $48 \mathrm{~h}$ of Febrifugine treatment relative to bees consuming sucrose syrup alone (Figure $5 \mathrm{~d}$ ), while the level of the reference gene $(\beta$-actin) was not altered by treatment (Figure 5e).

\section{DISCUSSION}

Components of the AAR are conserved in the honey bee, but not in $N$. ceranae. The absence of a recognizable AAR in $N$. ceranae and other microsporidia species might be expected, as microsporidia do not possess the biosynthetic pathways required to synthesize most amino acids and must import amino acids from the host cell through the use of transporters (Nakjang et al. 2013). However, the apparent conservation of Ser51, which is phosphorylated in yeast to regulate translation (Dever et al. 2016), suggests that novel mechanisms may be involved in regulating translation in this species despite the absence of homologs of kinases predicted to phosphorylate eIF2. In the absence of a canonical AAR pathway, it is interesting to speculate about whether microsporidia respond to amino acid limitation in host cells during infection. The impact of $N$. ceranae infection on honey bee health and productivity is still debated, and regional and strain-specific differences may complicate these issues (Goblirsch 2017; Martín-Hernández et al. 2018). When treated, N. ceranae infection of honey bee colonies in the USA has traditionally been controlled with Fumagillin. The future of 
a

음

d

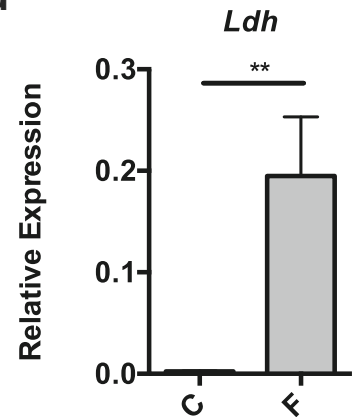

b

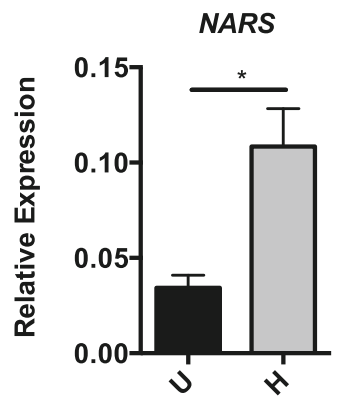

e

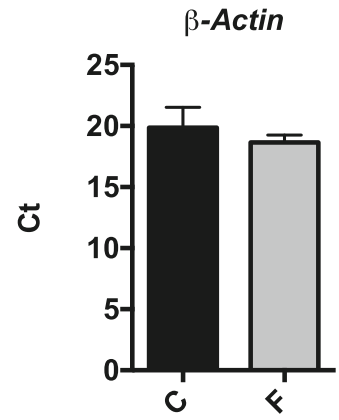

Figure 5. Honey bee AAR target genes are induced by Halofuginone and Febrifugine. Transcript levels of AAR target genes lactate dehydrogenase $(L d h)$ (a) and asparaginyl-tRNA synthetase gene (NARS) (b ) relative to $\beta$-actin in midgut tissue from individual bees with $(\mathrm{H}, n=4)$ or without $(\mathrm{U}, n=4) 200-\mu \mathrm{M}$ Halofuginone treatment after $48 \mathrm{~h}$. Threshold cycle number for $\beta$-actin from these bees (c). Transcript levels of AAR target gene $L d h$ (d) relative to $\beta$-actin in midgut tissue from individual bees with (F, $n=8)$ or without $(\mathrm{U}, n=8) 200-\mu \mathrm{M}$ Febrifugine treatment after $48 \mathrm{~h}$. Threshold cycle number for $\beta$-actin from these bees (e). Data is represented as Mean \pm SEM. Statistical significance is noted as $* p<0.05$ and $* * p<0.01$.

this drug is uncertain, making the discovery of novel therapeutic agents of significant importance. Eukaryotic pathogens can be challenging to combat using chemical antibiotics due to the phylogenetic closeness with their hosts, and attempts to find other treatments have not yet resulted in effective alternatives (reviewed in (Holt and Grozinger 2016)). However, a number of recent studies have found that compounds targeting members of the AARS family within eukaryotic pathogens can be highly efficacious treatment options for eukaryotic infectious organisms that cause disease in humans and livestock (reviewed in (Pham et al. 2014; Khan 2016)). For example, Halofuginone acts by inhibiting the prolyl-tRNA synthetase protein, which attaches proline amino acids to their cognate tRNA molecules (Keller et al. 2012; Zhou et al. 2014).
Halofuginone is a promising antimalarial drug (Herman et al. 2015) and has been used in veterinary medicine as a coccidiostat (Zhang et al. 2012) and appears to have activity against Cryptosporidium parvum (Trotz-Williams et al. 2011) and Toxoplasma gondii (Jain et al. 2015).

These studies showed that $N$. ceranae intensity was effectively reduced by treatment with Halofuginone. However, longer survival assays with bees revealed that the drug is toxic to bees at the concentrations most effective at killing N. ceranae. While Halofuginone has been found to be less toxic than Febrifugine in mammals, Febrifugine is equally potent in killing $N$. ceranae with less apparent toxicity to bees than Halofuginone. However, these results also show that at higher doses, toxicity of Febrifugine to bees is dramatically increased. Interestingly, the 
dose required for reducing infection intensity was higher in newly eclosed bees than in the landing board bees, perhaps suggesting age-related factors that impact efficacy of this drug against $N$. ceranae infection. Taken together, the results described here likely represent promising but early steps in using prolyl-tRNA synthetase inhibition as an anti-Nosema strategy. For example, novel Febrifugine derivatives have been developed (Kikuchi et al. 2006) with increased efficacy in killing multiple eukaryotic pathogens (Jain et al. 2017). Further, multiple groups have explored the key amino acids involved in binding of Halofuginone to Plasmodium prolyl-tRNA synthetase (PRS) and its inhibition (Jain et al. 2015; Herman et al. 2015). Further work could be done with the relevant prolyl-tRNA synthetases to pursue rational drug design to modify the base molecule Febrifugine to develop derivatives with a higher relative affinity to $N$. ceranae prolyl-tRNA synthetase than to the honey bee EPRS, leading to a better efficacy to toxicity ratio. However, the impacts of Febrifugine (or other derivatives) on different life stages and on the colony as a whole will need to be rigorously field tested before they can be used in the management of Nosema infection (Holt and Grozinger 2016). Thus, a more comprehensive analysis of the doses and long-term effects of such a treatment strategy on honey bee health, garnered through rigorous field trials, is imperative.

A number of additional points need to be considered in evaluating tRNA-synthetase inhibitors as drugs for use to control $N$. ceranae infection in honey bees. Resistance to Halofuginone does occur in malaria parasites (Herman et al. 2014; Herman et al. 2015) through homeostatic increase in proline synthesis machinery and mutation or amplification of the PRS gene. Changes in the $P R S$ are the most likely pathway to potential resistance in $N$. ceranae. As no AAR machinery or proline metabolic enzymes are evident in $N$. ceranae, alterations in proline synthesis are unlikely. However, microsporidia do possess a significant number of transporters for amino acids (Nakjang et al. 2013), and this is likely how proline is acquired by the parasitic cell. Therefore, mechanisms to increase host proline synthesis or microsporidia proline transport could change sensitivity to these compounds. In a related point, a recent study showed that $N$. ceranae loads eclipsed pre-treatment levels after cessation of Fumagillin treatment through an unknown mechanism (Huang et al. 2013). Thus, future studies to examine the stability of suppression should also be undertaken.

Microsporidia infect diverse invertebrate species playing important roles throughout our food production system, including many types of beneficial invertebrates such as pollinators (Weiss and Becnel 2014). In addition to insects, microsporidian infections are also problematic in other agriculturally important species. In the aquaculture industry, microsporidia are a significant source of pathology in multiple fish species, leading to loss of productivity (Weiss and Becnel 2014). Microsporidia also cause significant losses among aquatic invertebrates, such as crustaceans, that represent an important food and income source for humans (Weiss and Becnel 2014). Microsporidian infection is also a health issue for humans. While not a major problem in healthy individuals, immunocompromised individuals are highly susceptible to infection by a variety of microsporidian parasites, such as Encephalitozoon species (Weiss and Becnel 2014). Most microsporidia species that cause disease in agriculturally important animals and humans are predicted to possess PRS proteins that bind Halofuginone (Suppl Table 3). Thus, the results from these studies might be applicable in agriculture and human health.

Amino acid deprivation or AARS inhibition can be considered to negatively affect proteostasis, the homeostasis of protein synthesis, folding, function, and degradation both within a cell and in an organism as a whole (Klaips et al. 2017). A number of normal and pathologic conditions can lead to disruption of proteostasis, which is in turn sensed by a number of signaling systems in the cell. Comparison of the AAR of honey bees of those in other invertebrates, such as the fly (Santoyo et al. 1997; Olsen et al. 1998) and nematode (Nukazuka et al. 2008), suggests that it is quite similar in form and function. Interestingly, GCN2 and the AAR pathway have been linked to metabolism, development, and aging in these organisms (for example, see (Kang et al. 2015; 
Kang et al. 2017)), suggesting that these data will help add to our understanding of honey bee cellular stress responses and honey bee health.

Febrifugine is also found in the common garden plants of the genus Hydrange a (Ishih et al. 2006), which do serve as a nectar source for honey bees (Suppl Figure 4). Animals are known to employ self-medication or zoopharmacognosy (de Roode et al. 2013) and many invertebrates (Schmid-Hempel 2017), including honey bees (Erler and Moritz 2016), and participate in this behavior. Honey bees infected with $N$. ceranae prefer honey that possessed higher antibiotic activity (Gherman et al. 2014). Bumble bees ingest more nectar containing the antiparasitic compound anabasine when parasitized by a specific trypanosome (Anthony et al. 2015). While Febrifugine levels in nectar are unknown (Ishih et al. 2006), it is tempting to speculate that honey bees may preferentially consume Hydrangea nectar when parasitized by microsporidia.

In summary, perturbing the cellular process linked to the AAR through pharmacological inhibition of prolyl-tRNA synthetase activity results in reduction in $N$. ceranae infection intensity. Evident toxicity to bees suggests further work is required to make this approach safe and feasible. However, these results may provide proof of principle that tRNA synthetase inhibition could eventually be an effective treatment and suggest that targeting cellular stress pathways is a promising strategy for identification of potential novel therapeutics.

\section{ACKNOWLEDGEMENTS}

J.W.S. is thankful to Project Apis m. for their generous support in completion of this project.

L'inhibition de la prolyl-tRNA synthétase réduit l'intensité de l'infection par les microsporidies chez les abeilles.

Nosema ceranae / microsporidia / abeille domestique / infection / ARNt synthétase.
Die Hemmung der Prolyl-tRNA-Synthetase reduziert den Infektionsgrad mit Mikrosporidien bei Honigbienen.

Nosema ceranae / Mikrosporidien / Honigbienen / Infektion / tRNA-Synthetase.

\section{REFERENCES}

Anthony W.E., Palmer-Young E.C., Leonard A.S. et al (2015) Testing Dose-Dependent Effects of the Nectar Alkaloid Anabasine on Trypanosome Parasite Loads in Adult Bumble Bees. PLoS ONE 10, e0142496. https://doi.org/10.1371/journal.pone.0142496

Cantwell G.E. (1970) Standard methods for counting nosema spores. Am Bee J 110, 222-223

de Roode J.C., Lefèvre T., Hunter M.D. (2013) SelfMedication in Animals. Science 340, 150-151. https://doi.org/10.1126/science. 1235824

Dever T.E., Kinzy T.G., Pavitt G.D. (2016) Mechanism and Regulation of Protein Synthesis in Saccharomyces cerevisiae. Genetics 203, 65-107. https://doi. org/10.1534/genetics.115.186221

Erler S., Moritz R.F.A. (2016) Pharmacophagy and pharmacophory: mechanisms of self-medication and disease prevention in the honeybee colony (Apis mellifera). Apidologie 47, 389-411. https://doi. org/10.1007/s13592-015-0400-Z

Evans J.D., Schwarz R.S. (2011) Bees brought to their knees: microbes affecting honey bee health. Trends Microbiol 19, 614-620. https://doi.org/10.1016/j. tim.2011.09.003

Fries I., Chauzat M.-P., Chen Y.P. et al (2013) Standard methods for Nosema research. Journal of Apicultural Research 52, 1-28. https://doi.org/10.3896 /IBRA.1.52.1.14

Gherman B.I., Denner A., Bobiș O. et al (2014) Pathogenassociated self-medication behavior in the honeybee Apis mellifera. Behav Ecol Sociobiol 68, 1777-1784. https://doi.org/10.1007/s00265-014-1786-8

Goblirsch M. (2017) Nosema ceranae disease of the honey bee (Apis mellifera). Apidologie 49, 131-150. https://doi.org/10.1007/s13592-017-0535-1

Goulson D., Nicholls E., Botías C., Rotheray E.L. (2015) Bee declines driven by combined stress from parasites, pesticides, and lack of flowers. Science 347, 1255957. https://doi.org/10.1126/science. 1255957

Guo M., Yang X.-L., Schimmel P. (2010) New functions of aminoacyl-tRNA synthetases beyond translation. Nat Rev Mol Cell Biol 11, 668-674. https://doi. org/10.1038/nrm2956

Herman J.D., Rice D.P., Ribacke U. et al (2014) A genomic and evolutionary approach reveals non-genetic drug 
resistance in malaria. Genome Biol 15, 413. https://oi.org/10.1186/s13059-014-0511-2

Herman J.D., Pepper L.R., Cortese J.F. et al (2015) The cytoplasmic prolyl-tRNA synthetase of the malaria parasite is a dual-stage target of febrifugine and its analogs. Science Translational Medicine 7, $288 \mathrm{ra} 77$. https://doi.org/10.1126/scitranslmed.aaa3575

Holt H.L., Grozinger C.M. (2016) Approaches and Challenges to Managing Nosema (Microspora: Nosematidae) Parasites in Honey Bee (Hymenoptera: Apidae) Colonies. Journal of Economic Entomology 109, 1487-1503. https://doi.org/10.1093/jee/tow103

Huang W.-F., Solter L.F., Yau P.M., Imai B.S. (2013) Nosema ceranae Escapes Fumagillin Control in Honey Bees. PLoS Pathog 9, e1003185. https://doi. org/10.1371/journal.ppat.1003185

Ishih A., Miyase T., Suzuki T. et al (2006) Seasonal variation in the content of a febrifugine and isofebrifugine alkaloid mixture in aerial parts of Hydrangea macrophylla var. Otaksa, with special reference to its antimalarial activity. J Nat Med 61, 213-216. https://doi.org/10.1007/s11418-006-0124-5

Jain V., Yogavel M., Oshima Y. et al (2015) Structure of Prolyl-tRNA Synthetase-Halofuginone Complex Provides Basis for Development of Drugs against Malaria and Toxoplasmosis. Structure 23, 819-829. https://doi.org/10.1016/j.str.2015.02.011

Jain V., Yogavel M., Kikuchi H. et al (2017) Targeting Prolyl-tRNA Synthetase to Accelerate Drug Discovery against Malaria, Leishmaniasis, Toxoplasmosis, Cryptosporidiosis, and Coccidiosis. Structure 25:14951505.e6. https://doi.org/10.1016/j.str.2017.07.015

Jiang S., Zeng Q., Gettayacamin M. et al (2005) Antimalarial activities and therapeutic properties of febrifugine analogs. Antimicrob Agents Chemother 49, 1169-1176. https://doi.org/10.1128 /AAC.49.3.1169-1176.2005

Johnston B.A., Hooks K.B., McKinstry M., Snow J.W. (2016) Divergent forms of endoplasmic reticulum stress trigger a robust unfolded protein response in honey bees. J Insect Physiol 86, 1-10. https://doi. org/10.1016/j.jinsphys.2015.12.004

Kanehisa M., Goto S. (2000) KEGG: kyoto encyclopedia of genes and genomes. Nucleic Acids Research 28, 27-30. https://doi.org/10.1093/nar/28.1.27

Kang K., Ryoo H.D., Park J.-E. et al (2015) A Drosophila Reporter for the Translational Activation of ATF4 Marks Stressed Cells during Development. PLoS ONE 10, e0126795. https://doi.org/10.1371/journal. pone.0126795.s005

Kang M.-J., Vasudevan D., Kang K. et al (2017) 4E-BP is a target of the GCN2-ATF4 pathway during Drosophila development and aging. The Journal of Cell Biology 216, 115-129. https://doi.org/10.1083/jcb.201511073. dv

Keller T.L., Zocco D., Sundrud M.S. et al (2012) Halofuginone and other febrifugine derivatives inhibit prolyl-tRNA synthetase. Nature Chemical Biology $\mathbf{8}$, 311-317. https://doi.org/10.1038/NCHEMBIO.790
Khan S. (2016) Recent advances in the biology and drug targeting of malaria parasite aminoacyl-tRNA synthetases. Malar J 15, 1-10. https://doi.org/10.1186 /s12936-016-1247-0

Kikuchi H., Yamamoto K., Horoiwa S. et al (2006) Exploration of a New Type of Antimalarial Compounds Based on Febrifugine. J Med Chem 49, 4698-4706. https://doi.org/10.1021/jm0601809

Klaips C.L., Jayaraj G.G., Hartl F.U. (2017) Pathways of cellular proteostasis in aging and disease. The Journal of Cell Biology 217, 51-63. https://doi.org/10.1083 /jcb.201709072

Lee S.C., Corradi N., Doan S. et al (2010) Evolution of the sex-Related Locus and Genomic Features Shared in Microsporidia and Fungi. PLoS ONE 5, e10539. https://doi.org/10.1371/journal.pone.0010539.s005

Lee J.E., Oney M., Frizzell K. et al (2015) Drosophila melanogaster activating transcription factor 4 regulates glycolysis during endoplasmic reticulum stress. G3 (Bethesda) 5, 667-675. https://doi.org/10.1534 /g3.115.017269

Martín-Hernández R., Bartolomé C., Chejanovsky N. et al (2018) Nosema ceranaein Apis mellifera: a 12 years postdetection perspective. Environmental Microbiology 20, 1302-1329. https://doi.org/10.1111/14622920.14103

Mendoza Y., Diaz-Cetti S., Ramallo G. et al (2017) Nosema ceranae Winter Control: Study of the Effectiveness of Different Fumagillin Treatments and Consequences on the Strength of Honey Bee (Hymenoptera: Apidae) Colonies. Journal of Economic Entomology 110, 15. https://doi.org/10.1093/jee/tow228

Nakjang S., Williams T.A., Heinz E. et al (2013) Reduction and Expansion in Microsporidian Genome Evolution: New Insights from Comparative Genomics. Genome Biology and Evolution 5, 2285-2303. https://doi. org/10.1093/gbe/evt184

Nukazuka A., Fujisawa H., Inada T. et al (2008) Semaphorin controls epidermal morphogenesis by stimulating mRNA translation via eIF2 in Caenorhabditis elegans. Genes Dev 22, 1025-1036. https://doi.org/10.1101/gad.1644008

Olsen D.S., Jordan B., Chen D. et al (1998) Isolation of the gene encoding the Drosophila melanogaster homolog of the Saccharomyces cerevisiae GCN2 eIF-2alpha kinase. Genetics 149, 1495-1509

Pham J.S., Dawson K.L., Jackson K.E. et al (2014) Aminoacyl-tRNA synthetases as drug targets in eukaryotic parasites. Int J Parasitol Drugs Drug Resist 4, 1-13. https://doi.org/10.1016/j.ijpddr.2013.10.001

Rose M.D., Winston F., Hieter P. (1990) Methods in Yeast Genetics: A Laboratory Course Manual (Plainview, New York). American Psychological Association, Washington

Santoyo J., Alcalde J., Méndez R. et al (1997) Cloning and characterization of a cDNA encoding a protein synthesis initiation factor-2 alpha (eIF-2 alpha) kinase from Drosophila melanogaster - Homology to yeast GCN2 protein kinase. J Biol Chem 272, 12544-12550. https://doi.org/10.1074/jbc.272.19.12544 
Schmid-Hempel P. (2017) Parasites and Their Social Hosts. Trends Parasitol 33, 453-462. https://doi.org/10.1016 j.pt.2017.01.003

Snow J.W., Ceylan Koydemir H., Karinca D.K. et al (2019) Rapid imaging, detection, and quantification of Nosema ceranae spores in honey bees using mobile phone-based fluorescence microscopy. Lab on a Chip 19, 789-797. https://doi.org/10.1039/C8LC01342J

Trotz-Williams L.A., Jarvie B.D., Peregrine A.S. et al (2011) Efficacy of halofuginone lactate in the prevention of cryptosporidiosis in dairy calves. Veterinary Record 168, 509-509. https://doi.org/10.1136/vr. d1492

van den Heever J.P., Thompson T.S., Curtis J.M. et al (2014) Fumagillin: An Overview of Recent Scientific Advances and Their Significance for Apiculture. J Agric Food Chem 62, 2728-2737. https://doi. org/10.1021/jf4055374
Weiss L.M., Becnel J.J. (2014) Microsporidia. John Wiley \& Sons, Chichester

Zhang D.-F., Sun B.-B., Yue Y.-Y. et al (2012) Anticoccidial effect of halofuginone hydrobromide against Eimeria tenella with associated histology. Parasitol Res 111, 695-701. https://doi.org/10.1007 /s00436-012-2889-7

Zhou H., Sun L., Yang X.-L., Schimmel P. (2014) ATPdirected capture of bioactive herbal-based medicine on human tRNA synthetase. Nature 494, 121-124. https://doi.org/10.1038/nature11774

Publisher's note Springer Nature remains neutral with regard to jurisdictional claims in published maps and institutional affiliations. 